\title{
Screening for optimal protease producing Bacillus licheniformis strains with polymer-based controlled-release fed-batch microtiter plates
}

Tobias Habicher ${ }^{1 \dagger}$, Tobias Klein ${ }^{2 \dagger}$, Jacqueline Becker ${ }^{2}$, Andreas Daub² and Jochen Büchs ${ }^{1 *}$ (D)

\begin{abstract}
Background: Substrate-limited fed-batch conditions have the favorable effect of preventing overflow metabolism, catabolite repression, oxygen limitation or inhibition caused by elevated substrate or osmotic concentrations. Due to these favorable effects, fed-batch mode is predominantly used in industrial production processes. In contrast, screening processes are usually performed in microtiter plates operated in batch mode. This leads to a different physiological state of the production organism in early screening and can misguide the selection of potential production strains. To close the gap between screening and production conditions, new techniques to enable fed-batch mode in microtiter plates have been described. One of these systems is the ready-to-use and disposable polymer-based controlledrelease fed-batch microtiter plate (fed-batch MTP). In this work, the fed-batch MTP was applied to establish a glucoselimited fed-batch screening procedure for industrially relevant protease producing Bacillus licheniformis strains.
\end{abstract}

Results: To achieve equal initial growth conditions for different clones with the fed-batch MTP, a two-step batch preculture procedure was developed. Based on this preculture procedure, the standard deviation of the protease activity of glucose-limited fed-batch main culture cultivations in the fed-batch MTP was $\pm 10 \%$. The determination of the number of replicates revealed that a minimum of 6 parallel cultivations were necessary to identify clones with a statistically significant increased or decreased protease activity. The developed glucose-limited fed-batch screening procedure was applied to 13 industrially-relevant clones from two B. licheniformis strain lineages. It was found that 12 out of 13 clones (92\%) were classified similarly as in a lab-scale fed-batch fermenter process operated under glucoselimited conditions. When the microtiter plate screening process was performed in batch mode, only 5 out of 13 clones (38\%) were classified similarly as in the lab-scale fed-batch fermenter process.

Conclusion: The glucose-limited fed-batch screening process outperformed the usual batch screening process in terms of the predictability of the clone performance under glucose-limited fed-batch fermenter conditions. These results highlight that the implementation of glucose-limited fed-batch conditions already in microtiter plate scale is crucial to increase the precision of identifying improved protease producing $B$. licheniformis strains. Hence, the fedbatch MTP represents an efficient high-throughput screening tool that aims at closing the gap between screening and production conditions.

Keywords: Screening, Fed-batch, Microtiter plate, Protease, High-throughput

*Correspondence: jochen.buechs@avt.rwth-aachen.de

${ }^{\dagger}$ Tobias Habicher and Tobias Klein contributed equally to this work

${ }^{1}$ RWTH Aachen University, AVT - Biochemical Engineering,

Forckenbeckstraße 51, 52074 Aachen, Germany

Full list of author information is available at the end of the article

\section{Background}

Proteases are enzymes that catalyze the cleavage of proteins into smaller polypeptides or single amino acids. This ability offers a broad range of applications in industrial processes and products [1]. Thus, proteases are 
highly relevant in the technical enzyme market, especially in detergents [2]. Species from the genus Bacillus belong to the main producers of proteases. Bacillus species have high specific growth rates and the natural ability of secreting large amounts of proteins into the extracellular medium [3]. Nowadays, the production capabilities of protease producing Bacillus species are constantly improved by applying modern genetic engineering and molecular biology techniques [4-6]. With such techniques, large clone libraries are generated. Therefore, extensive screening campaigns have to be applied in order to identify strains with improved performance.

Screening of large clone libraries makes the use of small-scale high-throughput cultivation systems inevitable. The most frequently used small-scale cultivation systems for high-throughput screening applications are shaken bioreactors, such as microtiter plates $[7,8]$. Microtiter plates are cost-efficient and have a simple and functional design, which enables a high degree of parallelization and automation $[9,10]$. Basic correlations for mass transfer and power input further improved the understanding of microtiter plates [7]. However, microtiter plates were initially designed to be operated in batch mode. In contrast, the predominant mode in industrial protease production processes is fed-batch mode [2]. It is well known that with fed-batch mode, batch-related effects, such as overflow metabolism, osmotic inhibition and substrate inhibition, can be prevented [11]. Further, fed-batch mode allows to prevent catabolite repression. The prevention of catabolite repression plays an essential role when using promoters of genes related to catabolism. Catabolite controlled promoters are considered to be strong, and thus, commonly used with Bacillus species in industrial applications $[4,6,12]$. The induction and repression of these promoters, however, is directly coupled to the availability of the preferred carbon source, which most often is glucose. Thus, high glucose concentrations, as they occur in batch mode, have a repressive effect on protease formation [13].

It was demonstrated by transcriptome and proteome analyses that under batch and fed-batch conditions B. licheniformis cells are subject to completely different physiological conditions $[14,15]$. The importance of avoiding such different physiological conditions during screening and production was already indicated by Tännler et al. [16]. Tännler et al. established a microtiter plate-based screening process for riboflavin producing Bacillus subtilis mutants with raffinose as carbon source. Raffinose mimicked, to some extent, carbon-limitation in fed-batch cultures, which enabled a more efficient identification of riboflavin overproducing mutants in comparison to batch conditions with glucose in excess. Scheidle et al. implemented a microtiter plate-based glucose release system to screen Hansenula polymorpha clones under glucose-limited fed-batch conditions [17]. The comparison of batch and fed-batch clone ranking revealed that clones were ranked completely differently in each mode. When comparing batch to glucose-limited fed-batch screening conditions with Pichia pastoris, similar results were found $[18,19]$. Thus, the likelihood to identify improved strains for fed-batch fermentation processes is increased if the screening process is performed in fed-batch mode as well.

To establish similar physiological conditions during screening and production, microtiter plate-based cultivation systems enabling fed-batch operation have been developed $[8,20,21]$. The BioLector ${ }^{\circledR}$ Pro (m2p-labs $\mathrm{GmbH}$, Baesweiler, Germany) applies a microfluidic chip on a 48-well microtiter plate to continuously transfer a substrate solution from a reservoir well into a culture well, enabling 32 parallel cultivations [22, 23]. The microMatrix (Applikon Biotechnology, Delft, Netherlands) has built-in micro-injection valves that continuously add a substrate solution to wells of a 24-well microtiter plate. Besides high investment and operational costs for the BioLector ${ }^{\circledR}$ Pro and the micro-Matrix, the possibility of feeding is coupled to the peripheral equipment of these devices. Thus, fed-batch cultivations in parallel microtiter plates are not feasible, which results in limited throughput or high investment costs.

Microtiter plate-based fed-batch systems that are independent of peripheral equipment are enzyme-based and polymer-based glucose auto-delivery systems. The EnBase ${ }^{\circledR}$ system (BioSilta, Oulu, Finnland) is based on the enzymatic degradation of starch, in which the glucose release rate can be adjusted via the enzyme concentration [24]. Thus, the EnBase ${ }^{\circledR}$ system is independent of scale and highly parallelizable, allowing high-throughput operation. However, the enzymatic system was found to be sensitive against changing cultivation conditions, such as $\mathrm{pH}$ and temperature [25]. Furthermore, many organisms, including Bacillus species, secrete amylases and proteases, leading to uncontrollable glucose release kinetics. An alternative glucose auto-delivery system is the fed-batch MTP (Feed Plate ${ }^{\circledR}$, Kuhner shaker GmbH, Herzogenrath, Germany). It has a solid silicone matrix with embedded glucose crystals immobilized on the bottom of each well (Fig. 1). It is ready-to-use, disposable and parallelizable and thus applicable for high-throughput operation. In contrast to the EnBase ${ }^{\circledR}$ system, the fedbatch MTP was demonstrated to be less sensitive against changing cultivation conditions [26]. Furthermore, amylases and proteases do not impair glucose release. Nowadays, different fed-batch MTP formats, ranging from 24, 48 and 96 round- and square-wells, with different release characteristics are available. 


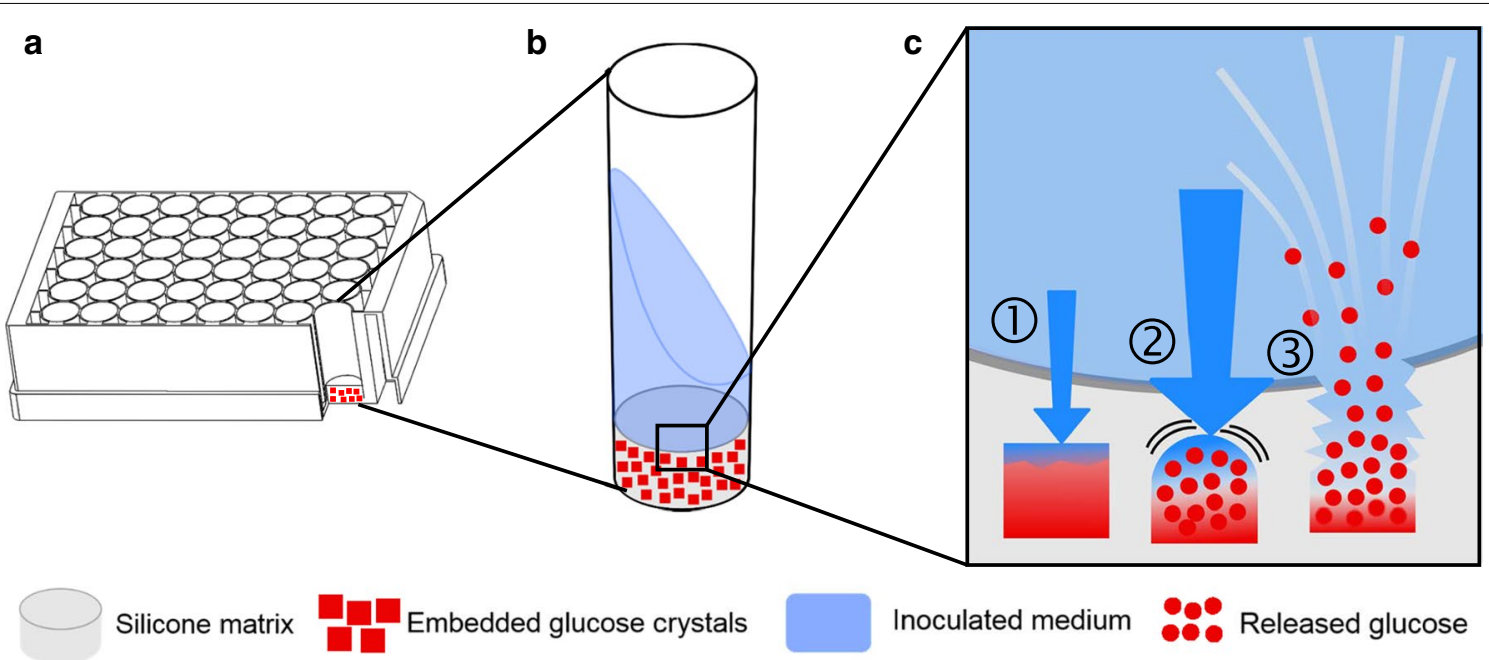

Fig. 1 Principle of the polymer-based controlled-release fed-batch microtiter plate (fed-batch MTP). a Fed-batch MTP with cross section view into a single well. $\mathbf{b}$ Close-up illustration of a single round-well showing the silicone matrix with embedded glucose crystals on its bottom. $\mathbf{c}$ Working principle of the polymer-based controlled-release system adapted from Keil et al. [26]. (1) Water diffuses into the silicone matrix and starts dissolving glucose crystals, thereby creating a highly concentrated glucose solution. (2) Due to the osmotic pressure, more water diffuses into the cavity. (3) Little cracks and channels are established in the silicone matrix, which is followed by the release of the glucose solution into the culture medium

In this study, the previously established fed-batch process for protease expression with B. licheniformis in fed-batch MTP's [27] was applied to perform a highthroughput and glucose-limited fed-batch screening campaign. The screening campaign was performed with a full set of $B$. licheniformis clones from two strain lineages without pre-selection (13 in total). The clone classification achieved under glucose-limited fed-batch conditions was compared to the clone classification achieved under batch conditions. Finally, the clone classification was validated with a lab-scale fed-batch fermenter operated under glucose-limited conditions. The presented microtiter plate-based glucose-limited fed-batch screening process aims at showing the advantage of establishing similar physiological conditions in microtiter plate screening and controlled fermenter processes in order to correctly identify protease producing $B$. licheniformis clones with improved performance.

\section{Results}

\section{Preculture development}

An often underestimated problem during screening processes is unequal growth of different clones in the main culture [28]. With unequal growth, individual clones are in uneven growth phases at the time of harvest, but are also exposed to the stationary phase for different lengths of time. This results in different clone rankings [29]. Variations in growth in the main culture are mainly caused by differences in the initial biomass concentration and in the duration of the lag-phase.
Since such differences are attributed to the inoculum, different preculture procedures were investigated and are presented in Fig. 2.

In Fig. 2a, inoculation was realized by directly transferring single colonies from LB agar plates into individual wells of a batch main culture. The biomass growth (scattered light intensity) of the batch main culture was monitored online. The scattered light intensities of the batch main culture highlight unreproducible growth kinetics of individually picked colonies (Fig. 2a). The culture of colony 20, for example, has the shortest lag-phase and reaches the stationary phase after $65 \mathrm{~h}$ (Fig. 2a, dashed black line). In contrast, the cultures of colony 10 and 11 show no growth after $65 \mathrm{~h}$ (Fig. 2a, solid black lines).

In Fig. 2b, inoculation of a batch main culture was realized by performing the following sequential preculture procedure: 1 . colony picking, 2 . first preculture in complex medium, 3. second preculture in mineral medium. The first preculture was inoculated by picking single colonies from an agar plate. The scattered light intensities (colony 1-4) reveal comparable growth kinetics with a negligible lag-phase and a plateau in the scattered light intensity starting at around $12 \mathrm{~h}$ of cultivation. The inoculum transfer into the second preculture was conducted at $16 \mathrm{~h}$.

The second preculture was inoculated with $1 \%(\mathrm{v} / \mathrm{v})$ of the first preculture. The scattered light intensities (1-4) of the second preculture exhibit a similar growth behavior of all colonies with a short lag-phase of $6 \mathrm{~h}$ (Fig. 2b, second preculture). The second preculture was harvested 


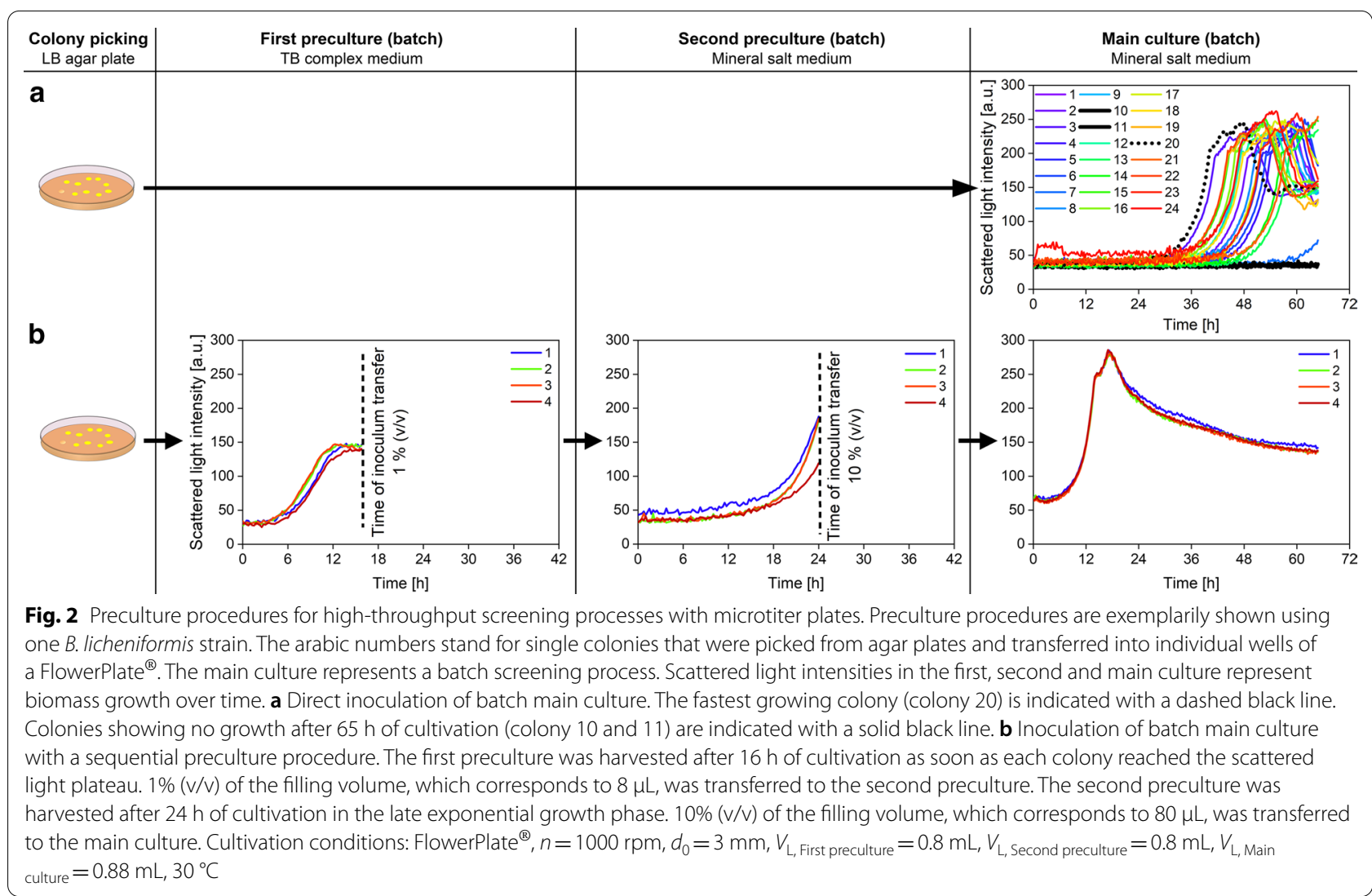

in the late exponential growth phase at $24 \mathrm{~h}$ (Fig. 2b, second preculture).

The batch main culture was inoculated with $10 \%(\mathrm{v} / \mathrm{v})$ of the second preculture. The scattered light intensities (1-4) of the batch main culture reveal synchronized growth of each colony with a negligible lag-phase (Fig. 2b, main culture). By means of the described preculture procedure, batch and fed-batch screening procedures were conducted as illustrated in Fig. 3.

\section{Determination of the number of replicates}

The number of replicates needed to obtain statistically significant screening results has to be determined before conducting high-throughput screening experiments. Factors that influence the number of replicates are the standard deviation of the performance indicator after repetitions, the performance difference that has to be detected (detectable difference) and the demanded statistical power. With the protease activity as performance indicator, the required number of replicates for highthroughput screening experiments with fed-batch MTP's used in this study was calculated. The results are depicted in Fig. 4.

In Fig. 4a, the final relative protease activities of glucose-limited fed-batch cultivations with fed-batch MTP's are presented. Glucose-limited fed-batch cultivations were realized in six wells $(n=6)$ with three individual plates (fed-batch MTP 1-3) using the same B. licheniformis strain. Each plate was inoculated according to the procedure presented in Fig. 3. Relative protease activities of cultivations with fed-batch MTP's (fed-batch MTP 1-3) show no significant difference on a significance level of $5 \%$ (Fig. $4 \mathrm{a}$ ). The highest standard deviation of the relative protease activity within one plate is $9.9 \%$ (Fig. $4 \mathrm{a}$, fed-batch MTP 3). Thus, the deviation of repetitive cultivations with fed-batch MTP's is in the range of $\pm 10 \%$.

In order to calculate the number of replicates, the value for the statistical power has to be selected. For the high-throughput screening in microtiter plates, a statistical power of 0.8 was chosen. Figure $4 \mathrm{~b}$ shows the number of replicates as a function of the standard deviation and the detectable difference with a constant statistical power of 0.8. The number of replicates increases with an increasing standard deviation as well as with a decreasing detectable difference.

In order to calculate the minimal number of replicates, the value of the minimal detectable difference was chosen with $\pm 15 \%$. With known minimal detectable difference of $\pm 15 \%$, standard deviation of $\pm 10 \%$ and a statistical power of 0.8 , the resulting minimal number of replicates 


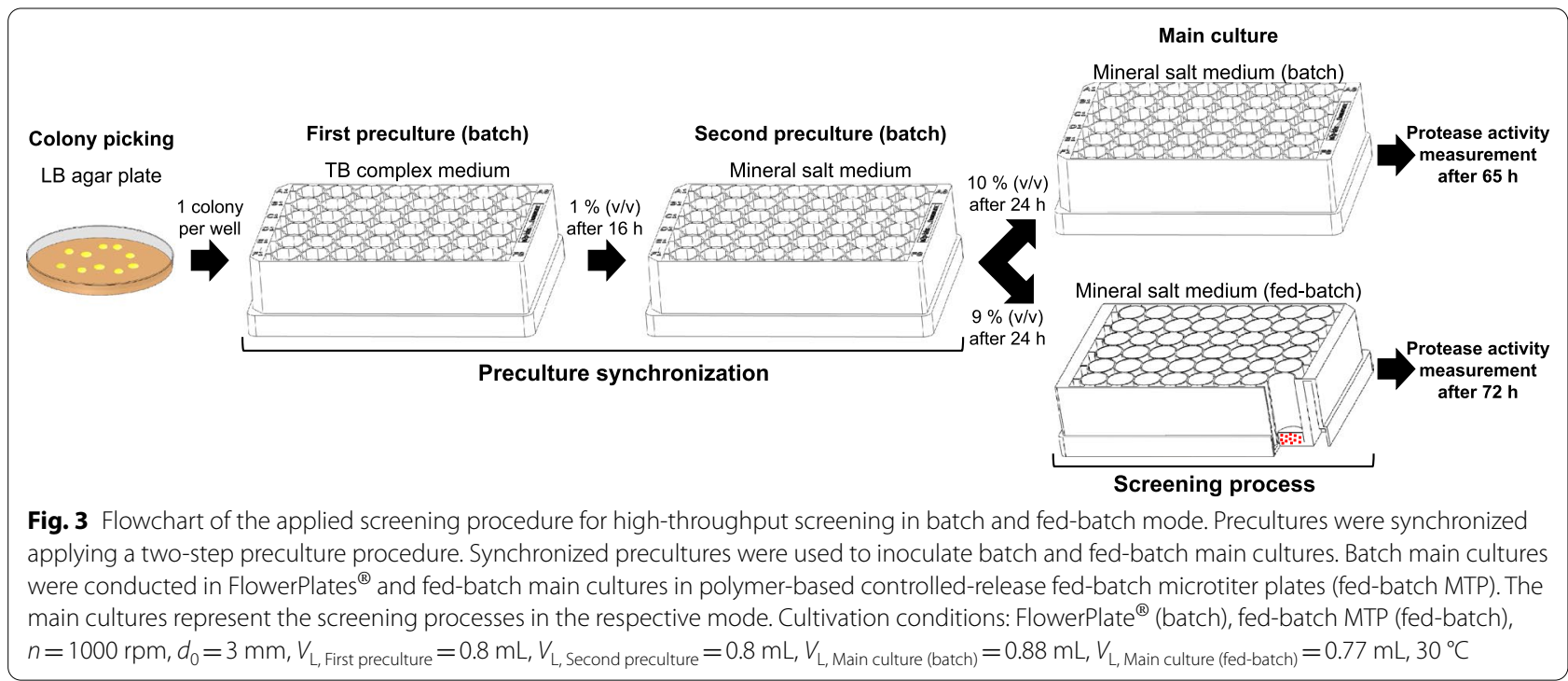

is 6 (Fig. 4b). In other words, a minimum of 6 parallel glucose-limited fed-batch cultivations have to be conducted on a fed-batch MTP as used in this study to detect a clone having a $15 \%$ increased or decreased protease activity with a statistical power of 0.8 .

\section{Comparison of batch and fed-batch screening}

B. licheniformis clones of two different lineages (\#1 and \#2) were screened under batch and glucose-limited fedbatch conditions according to the procedure presented in Fig. 3. Screening results were validated with a lab-scale fermenter operated under glucose-limited fed-batch conditions. The results of the microtiter plate (MTP)-based batch (batch MTP) and fed-batch screening (fed-batch MTP) as well as the experimental validation in the labscale fed-batch fermenter (fermenter) are summarized in Fig. 5.

Figure $5 \mathrm{a}$ and $\mathrm{b}$ depict the screening and fermenter results of the clone library of lineage \#1 and \#2, respectively. Based on these results, clones are classified into higher, lower or similar (no significant difference) performing clones relative to the control strain (Fig. 5c). The classification shown in Fig. $5 \mathrm{c}$ reveals that clones are classified differently with the batch MTP, the glucose-limited fed-batch MTP and the glucose-limited fed-batch process in a fermenter. The important question is, however, whether clones screened in batch mode (batch MTP) or fed-batch mode (fed-batch MTP) are classified similarly to the fed-batch fermenter process that mimics production conditions. Therefore, the clone classification of the batch screening (batch MTP) was compared to the classification achieved with the fed-batch fermenter process (fermenter). The comparison exhibits that the performance of 5 out of 13 clones is correctly predicted in batch screening mode, which corresponds to a predictability of $38 \%$. The comparison of the clone classification achieved with the fed-batch screening (fed-batch MTP) and fed-batch fermenter process (fermenter) reveals that 12 out of 13 clones are classified similarly. This corresponds to a correct prediction of $92 \%$.

\section{Discussion \\ Preculture development}

Assuming that the main culture shown in Fig. 2a represents a typical batch screening process that is terminated after $65 \mathrm{~h}$, individual clones are harvested in completely different growth phases. One reason for unequal growth behavior in the main culture relates to the colony picking process. Colony picking causes a transfer of different colony volume and the picked colonies can be in unequal physiological stages [28]. These variations cannot be avoided by changing the picking method (by hand or by picking robot) or picking instrument (inoculation loop, toothpick, pipet tip, etc.) $[29,30]$. However, although the colony picking method described in Fig. $2 \mathrm{~b}$ was similar to the procedure in Fig. 2a, scattered light intensities (1-4) of the first preculture exhibit negligible lag-phases. The prevention of the lag-phases can be attributed to the applied culture medium. In Fig. 2a, colonies from complex LB agar plates were directly transferred into mineral salt medium of the main culture. The changing substrate availability from complex to mineral basis results in extended lag-phases and unpredictable growth kinetics. Besides the substrate source, also physical changes within the culture environment, as for example the medium osmolality, influence growth of B. licheniformis [31, 32]. 

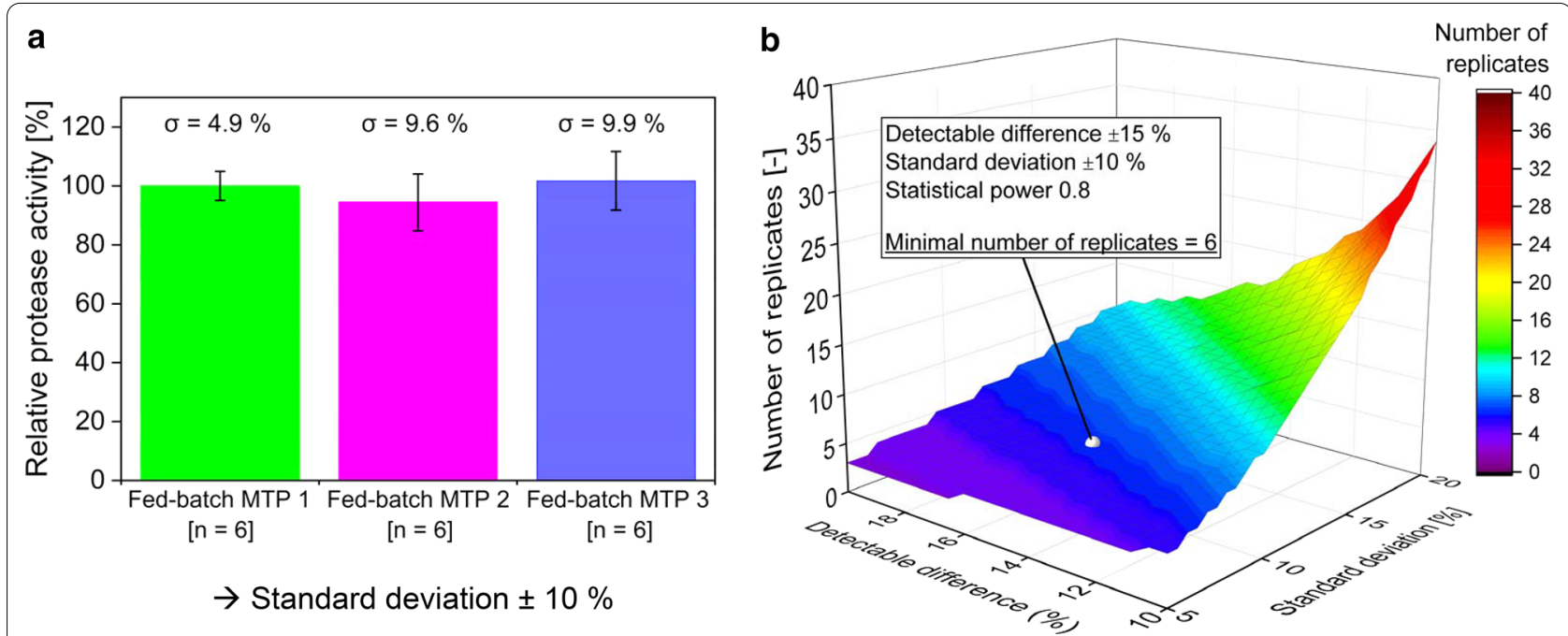

Fig. 4 Statistical approach to determine the minimal number of replicates for a fed-batch screening campaign. a Mean relative protease activity of fed-batch cultivations with three individual fed-batch MTP's (fed-batch MTP 1-3). The same B. licheniformis strain was used for all cultivations. The mean protease activity of fed-batch MTP 1 was normalized to 100\%. Protease activities of fed-batch MTP 2 and 3 were set in relation to fed-batch MTP 1. Error bars symbolize the standard deviation $(n=6)$. An univariate ANOVA $(F(215)=1.21, p=0.33)$ showed that there is no significant difference $(\alpha=5 \%)$ between the investigated plates. Cultivation conditions: fed-batch MTP, $n=1000 \mathrm{rpm}, d_{0}=3 \mathrm{~mm}, V_{\mathrm{L}, \text { Main culture (fed-batch) }}=0.77 \mathrm{~mL}$, $30^{\circ} \mathrm{C}$. $\mathbf{b}$ Number of replicates as a function of the detectable difference and standard deviation. The required detectable difference and statistical power are stated within the figure. The number of replicates was calculated with the MATLAB function sampsizepwr with a two-tailed $t$-test (normally distributed) as test type

By applying the sequential preculture procedure (Fig. 2b), colonies from complex LB agar plates were transferred into complex TB medium of the first preculture. Hence, the exemplarily shown cultivations using $\mathrm{TB}$ medium reveal efficient growth within the first preculture.

The positive effect on growth is not the only reason for using complex TB medium within the first preculture. Apart from the complex compounds, TB medium is supplemented with glycerol. In TB medium, glycerol represents the primary carbon source for B. licheniformis. The depletion of glycerol is indicated by a plateau of the scattered light intensity at approximately $12 \mathrm{~h}$ of cultivation (Fig. 2b, first preculture). At this point, the complex compounds act as slowly accessible substrate sources, which prevent $B$. licheniformis from undergoing carbon starvation. This results in equal growth conditions with equal biomass concentrations for each individually picked colony, which is indicated by the scattered light plateau (colony 1-4) reached after $16 \mathrm{~h}$ of cultivation (Fig. 2b, first preculture, Additional file 1). If B. licheniformis cultures face carbon starvation, the scattered light intensity exhibits a declining trend instead of showing a plateau (Fig. 2b, main culture; Additional files 1 and 2). Under carbon starvation, Bacillus cells exhibit cell lysis [33, 34] and morphological changes [35], resulting in decreasing scattered light intensities [36].
The reason for using a relatively small inoculum volume of $1 \%(\mathrm{v} / \mathrm{v})$ to inoculate the second preculture was to minimize the transfer of unmetabolized complex compounds (Fig. 2b, second preculture). The short lag-phase is most probably caused by transferring the culture from complex to mineral medium. Diluting the complex compounds is not the only reason for introducing a second preculture. The second preculture also serves to adapt the $B$. licheniformis culture to the mineral salt medium, which is also applied in the main culture. However, when working with mineral salt media in the preculture, the time of inoculum transfer determines the inoculum quality. Since the mineral salt medium contains $20 \mathrm{~g} / \mathrm{L}$ glucose as only carbon source, glucose depletion results in carbon starvation. Besides cell lysis [33, 34] and morphological changes [35], carbon starvation causes a variety of metabolic changes in Bacillus cultures [15, 37, 38]. To prevent the culture from undergoing these changes, the second preculture was harvested in the late exponential growth phase at $24 \mathrm{~h}$ (Fig. $2 \mathrm{~b}$, second preculture), prior to glucose depletion.

The batch main culture was inoculated with $10 \%(\mathrm{v} / \mathrm{v})$ of the second preculture to ensure a high initial biomass concentration (Fig. 2b, main culture). This is of particular importance when the screening process is conducted with the fed-batch MTP. Due to the release principle of this microtiter plate (Fig. 1), glucose is released from the 


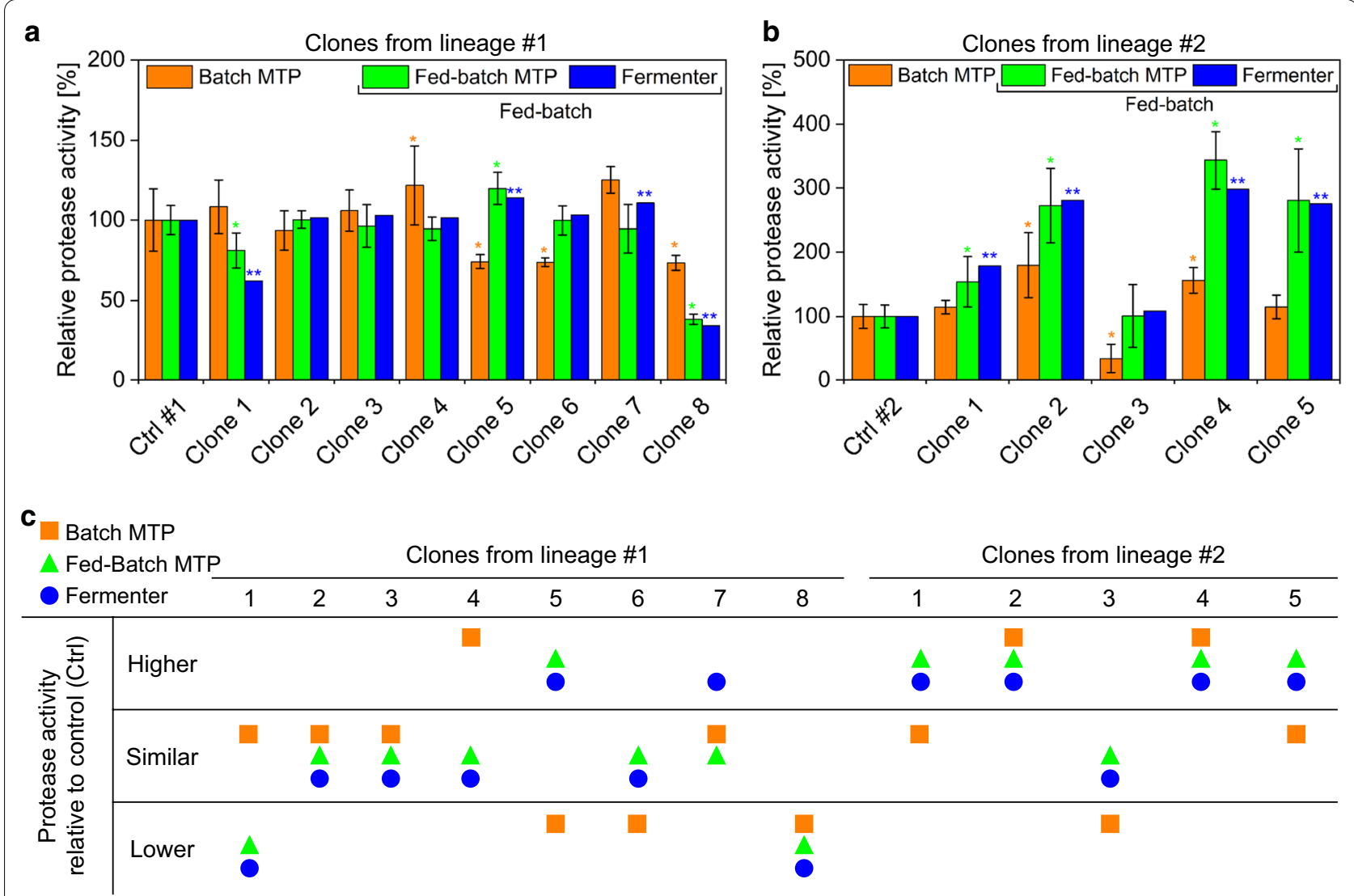

Fig. 5 High-throughput screening of B. licheniformis clones in batch and fed-batch mode. a Mean relative protease activity of B. licheniformis clones of strain lineage \#1 and $\mathbf{b}$ strain lineage \#2 screened with microtiter plates (MTP) in batch (batch MTP) and fed-batch mode (fed-batch MTP) and validated with a lab-scale fed-batch fermenter under glucose-limited conditions (fermenter). The mean protease activities of the control strain of lineage one ( $\mathrm{Ctrl} \# 1)$ and two ( $\mathrm{Ctrl} \# 2$ ) were normalized to 100\%. Protease activities of clones were set in relation to the corresponding control strain cultivated under identical conditions. Error bars symbolize the standard deviation (batch MTP and fed-batch MTP, $n=6$ ). Despite $n \geq 3$, no error bars are shown for the lab-scale fed-batch fermenter (fermenter). Significant difference between each clone and control strain (two-tailed $t$-test with equal variance) is indicated with * for $p<0.1$ and with ** for $p<0.05$. c Performance classification of clones screened in batch (batch MTP) and fed-batch mode (fed-batch MTP) with validation under glucose-limited fed-batch conditions in a lab-scale fermenter (fermenter). The term "Similar" implies that there is no significant difference

beginning of the cultivation (Additional file 3) [26, 27]. At this point, the biomass concentration is too low to consume all of the released glucose. This results in an initial batch phase. Its length is dependent on the initial biomass concentration [27]. Low initial biomass concentrations might cause an extended batch phase with oxygen limitation and overflow metabolism.

A commonly applied preculture procedure consists of a single preculture instead of a two-step procedure (Additional file 1). This single preculture can be performed either in complex medium or mineral salt medium. As described above, directly transferring picked colonies into mineral salt medium revealed substantial lagphases with unequal growth (Fig. 2a), which does not result in synchronized cultures. Precultures performed in complex medium showed negligible lag-phases and equal growth, thereby enabling growth synchronization (Fig. 2b, Additional file 1). However, when performing a single preculture in complex medium, complex compounds are transferred into the main culture. At the same time, a short lag-phase was observed when transferring the culture from the complex medium to the mineral salt medium (Fig. 2b). Both, the transfer of complex compounds and lag-phases become crucial when working with fed-batch MTP's. As mentioned above, the fed-batch MTP's require high initial cell concentrations. Thus, with a single preculture it is difficult to find a trade-off between reaching high initial cell densities and reducing the amount of transferred complex compounds into the main culture. By introducing a second preculture with mineral salt medium, the complex compounds transferred to the main culture can be reduced, on the 
one hand, and the B. licheniformis culture can adapt to the mineral salt medium, on the other, which enables equal growth with negligible lag-phases in the main culture (Fig. 2b).

Unequal growth conditions can also be prevented with the use of a robotic platform (RoboLector ${ }^{\circledR}$ ) consisting of a BioLector ${ }^{\circledR}$ and a liquid-handling robot $[9,39]$. Applying fed-batch mode to synchronize growth of precultures is another functional and well-described procedure [28, 29]. However, with the used B. licheniformis strain, two sequential preculture cultivations in batch mode represent an easy to implement and efficient solution to synchronize the precultures.

\section{Determination of the number of replicates}

In every high-throughput screening there is a trade-off between the number of replicates used for each strain and the number of different strains investigated. While the reliability increases with more replicates, the throughput decreases at the same time. Determination of the minimal number of replicates needed for a statistically sound evaluation of strain performance can be used to find an optimum between statistical reliability and throughput. However, for calculating the minimal number of replicates the standard deviation, the minimal detectable difference and the statistical power is required.

The standard deviation of repetitive cultivations with fed-batch MTP's is in the range of $\pm 10 \%$ (Fig. 4a). This standard deviation represents the sum of individual deviations occurring throughout the entire screening procedure. This includes the analytical procedure of the protease activity measurement. However, the largest deviation derives from the glucose-limited fed-batch cultivation with the fed-batch MTP. It has been found that glucose release from fed-batch MTP's exhibits a mean coefficient of variation of 4.5\% [26]. This deviation in glucose release directly influences the final protease activity.

The results in Fig. $4 \mathrm{~b}$ show that besides the standard deviation, the detectable difference influences the number of replicates. The minimal detectable difference describes to which extend the protease activity must at least increase or decrease in order to be detected with the demanded statistical power. In contrast to the standard deviation, the minimal detectable difference is a parameter that cannot be determined experimentally. The value of the minimal detectable difference relies for example on previous experiences with strain improvement programs. Since the number of replicates decreases with increasing minimal detectable difference (Fig. 4b), a clone that has a change in protease activity $> \pm 15 \%$ is still securely detected with 6 replicates (statistical power $\geq 0.8$ ). If only 3 replicates would be used, as often found in literature, only strains with a productivity increase $> \pm 30 \%$ compared to the control strain could be identified with a standard deviation of $\pm 10 \%$ and a statistical power of 0.8 (Additional file 4a). On the other hand, using only 3 replicates but selecting for strains with $\pm 15 \%$ improvement at $\pm 10 \%$ standard deviation would result in a statistical power of only 0.3 (Additional file $4 \mathrm{~b}$ ).

The statistical power describes the probability of correctly rejecting the null hypothesis, i.e. correctly identifying clones with different protease activities. Therefore, the statistical power is an important measure regarding the reliability of screening results. The higher the statistical power, the higher the number of replicates needed to correctly identify clones with a different protease activity (Additional file 4b). In order to find a trade-off between statistical reliability and number of replicates (Additional file $4 \mathrm{~b}$ ), a statistical power of 0.8 was chosen for this study.

\section{Comparison of batch and fed-batch screening}

The comparison of the predictability of batch (38\%) and fed-batch screening mode (92\%) highlights that implementing fed-batch conditions is essential to predict the clone performance similarly to the fed-batch fermenter that mimics production conditions. The necessity of implementing fed-batch conditions during screening is exemplarily discussed based on the results achieved with clone 4 and 5 from lineage \#1 and clone 1 and 5 from lineage \#2 (Fig. 5c). Clone 4 from lineage \#1 exhibits a significantly higher protease activity under batch screening conditions (Fig. 5c, batch MTP). With the assumption that the screening process is only conducted in batch mode, clone 4 would be selected as a promising strain candidate and would be further investigated under lab-scale fed-batch conditions. Under these conditions, however, clone 4 shows no significantly higher protease activity and would be rejected (Fig. 5c, fermenter). Under fed-batch screening conditions, in contrast, clone 4 is correctly identified as a strain with no significantly higher protease activity (Fig. 5c, fed-batch MTP). Consequently, with a screening process conducted in fed-batch mode, the strain would not have been considered further, thereby saving time and costs. The results achieved with clone 5 from lineage \# 1 and clone 1 and 5 from lineage \#2 represent an opposite scenario (Fig. 5c). Due to the similar (non-significant) or significantly lower performance achieved under batch screening conditions (Fig. 5c, batch MTP), these clones would directly be rejected. However, under fed-batch screening conditions, these clones were correctly identified as clones with a significantly higher protease activity than the control strain (Fig. 5c, fed-batch MTP and fermenter). This scenario shows that optimal protease producing clones can remain undetected under batch screening conditions, due to unequal 
physiological conditions between screening and production processes.

\section{Conclusion}

High-throughput screening is crucial in strain optimization programs. Usually, the screening processes are performed with microtiter plates in batch mode. However, batch conditions can cause unfavorable phenomena that can be prevented by applying fed-batch conditions. Therefore, production processes are usually operated under glucose-limited fed-batch conditions. The fedbatch MTP used in this study and commercialized by Kuhner Shaker $\mathrm{GmbH}$ as Feed Plate ${ }^{\circledR}$ is capable of establishing such glucose-limited fed-batch conditions in microtiter plates by continuously releasing glucose into the medium.

It was demonstrated that direct inoculation with colonies picked from agar plates resulted in unequal growth within the main culture. Thus, a sequential preculture procedure starting with colony picking was developed. By applying a two-step preculture procedure, equal growth conditions were achieved within the main culture. Based on this preculture procedure, the standard deviation of repetitive experiments with the fed-batch MTP was investigated. It was found that the standard deviation of the final protease activity of repetitive glucose-limited fed-batch cultivations is in the range of $\pm 10 \%$. With known standard deviation of $\pm 10 \%$, chosen minimal detectable difference of $\pm 15 \%$ and statistical power of 0.8 , the minimal number of replicates was 6. Without calculating the number of replicates, there is the risk of randomly choosing a too low number of replicates. This could lead to a situation where a statistically sound identification of improved clones is not achievable. Thus, initially attaining some key numbers and adjusting the screening setup accordingly can drastically improve the success of a screening campaign.

The comparison of the batch and fed-batch screening underlines the necessity of implementing glucose-limited fed-batch conditions already during early strain screening campaigns. In batch mode, $38 \%$ of the investigated $B$. licheniformis clones were classified similarly to the lab-scale fed-batch fermenter operated under glucoselimited conditions. In contrast, when applying glucoselimited fed-batch conditions in the microtiter plate, 92\% of the investigated clones were classified similarly to the lab-scale fed-batch fermenter process. These results demonstrate that the performance of a potential production strain can only be evaluated if the physiology of the strain is comparable between screening and production conditions. For a production process relying on glucoselimited fed batch, the fed-batch MTP's used in this study proved to be capable of implementing such conditions.
Hence, the fed-batch MTP represents an efficient highthroughput fed-batch screening tool that aims at closing the gap between screening and production.

\section{Methods}

\section{Strains}

Protease producing Bacillus licheniformis clones were provided by BASF SE (Ludwigshafen am Rhein, Germany). A heterologous protease was produced belonging to the group of subtilisin proteases originating from Bacillus lentus [40]. Screening was performed with a full set of clones of two different $B$. licheniformis strain lineages, both expressing the same protease. No pre-selection was made regarding the choice of clones. The clone library of strain lineage one (\#1) consisted of eight clones (clone 1-8) and one control strain, which is referred to as Ctrl \#1. The clone library of strain lineage two (\#2) consisted of five clones (clone 1-5) and one control strain, which is referred to as Ctrl \#2. Both lineages derive from rational strain engineering and the fed-batch MTP system was used to evaluate the impact of these strain modifications in comparison to the respective parent strain.

\section{Media}

The chemicals applied for media preparation were of analytical grade and purchased from Sigma-Aldrich Chemie $\mathrm{GmbH}$ (Steinheim, Germany), if not stated otherwise.

Agar plates were used for colony picking and were based on complex lysogeny broth (LB) medium. The LB medium contained per liter: $10 \mathrm{~g}$ tryptone (Pancreatic digest from casein, Sigma-Aldrich Chemie GmbH, Steinheim, Germany), $10 \mathrm{~g} \mathrm{NaCl}$ and $5 \mathrm{~g}$ yeast extract (Biospringer, Maisons-Alfort, France). The $\mathrm{pH}$ was adjusted to 7.0 with $5 \mathrm{M} \mathrm{NaOH}$. Subsequently, $15 \mathrm{~g}$ of bacteriological agar were dissolved in $1 \mathrm{~L}$ of $\mathrm{LB}$ medium and sterilized by autoclaving $\left(121^{\circ} \mathrm{C}\right.$ for $\left.20 \mathrm{~min}\right)$. At a temperature of approximately $50{ }^{\circ} \mathrm{C}$ the $\mathrm{LB}$ agar was poured into plates.

The complex terrific broth (TB) medium was used for the first preculture and contained per liter: $10 \mathrm{~g}$ glycerol $\left(\mathrm{C}_{3} \mathrm{H}_{8} \mathrm{O}_{3}\right), 12 \mathrm{~g}$ tryptone (Pancreatic digest from casein, Sigma-Aldrich Chemie GmbH, Steinheim, Germany), $24 \mathrm{~g}$ yeast extract (Biospringer, Maisons-Alfort, France), $12.54 \mathrm{~g} \mathrm{~K}_{2} \mathrm{HPO}_{4}$ and $2.31 \mathrm{~g} \mathrm{KH}_{2} \mathrm{PO}_{4}$. After autoclaving $\left(121{ }^{\circ} \mathrm{C}\right.$ for $\left.20 \mathrm{~min}\right)$, the medium was stored at $4{ }^{\circ} \mathrm{C}$ for not longer than 6 months.

A mineral salt medium was used for the second preculture and for main cultures in microtiter plates. The mineral salts and trace elements contained in the mineral salt medium are described in $\mathrm{WO} / 2020 / 169,564$ [41]. The concentration of the respective chemicals was adapted to the lower amount of glucose that was used for batch and fed-batch cultivations compared to the $>200 \mathrm{~g} / \mathrm{L}$ of 
glucose added in the above mentioned patent application. To maintain $\mathrm{pH}$ in a reasonable range the medium contained $0.2 \mathrm{M}$ MOPS buffer and the initial $\mathrm{pH}$ was adjusted to 7.5. For batch and fed-batch cultivations in microtiter plates, the medium was supplemented with 20 and $0 \mathrm{~g} / \mathrm{L}$ glucose, respectively. Glucose release in fedbatch MTP's is shown in Additional file 3. Except for the glucose concentration, the mineral salt medium formulation was identical for batch and fed-batch cultivations with microtiter plates.

\section{Preculture}

The preculture procedure consisted of sequential steps and was started by plating glycerol cryo stocks on LB agar plates, followed by an overnight incubation at $30{ }^{\circ} \mathrm{C}$. Single colonies were picked with toothpicks from the LB agar plate and used to inoculate the first preculture. Each colony was then transferred into one well of a FlowerPlate $^{\circledR}$ (MTP-B48-B, m2p labs GmbH, Baesweiler, Germany) filled with $V_{\mathrm{L}}$, First preculture $=0.8 \mathrm{~mL}$ of complex TB medium. After $16 \mathrm{~h}, 1 \%(\mathrm{v} / \mathrm{v})$ of the culture broth of the first preculture $(8 \mu \mathrm{L})$ was used to inoculate the second preculture. The second preculture was performed in a FlowerPlate ${ }^{\circledR}$ with a filling volume $V_{\mathrm{L}}$, Second preculture $=0.8 \mathrm{~mL}$ using a mineral salt medium. After $24 \mathrm{~h}, 10 \%$ and $9 \%(\mathrm{v} / \mathrm{v})$ of the culture broth of the second preculture were used to inoculate the batch and fed-batch main culture, respectively. Cultivations with FlowerPlates ${ }^{\circledR}$ were carried out on a BioLector ${ }^{\circledR}$ device (m2p labs $\mathrm{GmbH}$, Baesweiler, Germany). The BioLector ${ }^{\circledR}$ device enabled online biomass monitoring via scattered light intensity at a wavelength $\lambda=620 \mathrm{~nm}$ (gain $=20$ ) (Fig. 2, Additional files 1 and 2). To reduce evaporation and prevent cross contamination, FlowerPlates ${ }^{\circledR}$ were sealed with a gaspermeable sealing foil (F-GPR48-10, m2p labs GmbH, Baesweiler, Germany). Cultivations were performed with a shaking frequency $n=1000 \mathrm{rpm}$, a shaking diameter $d_{0}=3 \mathrm{~mm}$, at $30^{\circ} \mathrm{C}$ and a relative humidity $\phi>75 \%$.

\section{Main culture}

Main cultures in microtiter plates were performed in batch and fed-batch mode using a mineral salt medium. Batch cultivations were performed in FlowerPlates ${ }^{\circledR}$ on a BioLector ${ }^{\circledR}$ device (m2p labs GmbH, Baesweiler, Germany) with a filling volume $V_{\mathrm{L} \text {, Main culture (batch) }}=0.88 \mathrm{~mL}$. Fed-batch cultivations were performed in a 48-roundwell fed-batch MTP's (Feed Plate ${ }^{\circledR}$, Art. Nr.: SMFP08004, Kuhner Shaker GmbH, Herzogenrath, Germany) on an orbital climo shaker (Multitron Pro, Infors AG, Bottmingen, Switzerland) with a filling volume $V_{\mathrm{L} \text {, Main culture }}$ (fed-batch) $=0.77 \mathrm{~mL}$. To reduce evaporation and prevent cross contamination, both FlowerPlates ${ }^{\circledR}$ and fed-batch MTP's were sealed with a gas-permeable sealing foil
(F-GPR48-10, m2p labs GmbH, Baesweiler, Germany). Cultivations were performed with a shaking frequency $n=1000 \mathrm{rpm}$, a shaking diameter $d_{0}=3 \mathrm{~mm}$, at $30^{\circ} \mathrm{C}$ and a relative humidity $\phi>75 \%$. The cultivation conditions in microtiter plate-based batch and fed-batch main cultures were adjusted so that an oxygen limitation was prevented (Additional file 2) [27]. Culture acidification was prevented by using MOPS buffer, thereby keeping the $\mathrm{pH}$ in the neutral range between 7 and 7.5 (Additional file 2) [27]. Batch and fed-batch cultivations were terminated after 65 and $72 \mathrm{~h}$, respectively. The harvested culture broth was filtered $(0.45 \mu \mathrm{m}$ filter $)$ and the protease activity analyzed. Figure 3 gives an overview of the applied screening procedure.

The fed-batch fermenter process was conducted in a lab-scale stirred tank reactor system (DASGIP ${ }^{\circledR}$ Bioblock $1 \mathrm{~L}$ fermentation system, Eppendorf AG, Jülich, Germany). The system was operated with a glucose feeding strategy resulting in glucose-limited fed-batch conditions. $\mathrm{pH}$ was controlled above 7 by addition of ammonia solution $\left(\mathrm{NH}_{4} \mathrm{OH}\right)$. The dissolved oxygen tension (DOT) was controlled at a setpoint of $30 \%$. The fermentation process has been described in detail in $\mathrm{WO} / 2020 / 169,564$ [41].

\section{Protease assay}

Protease activity measurement was based on the method developed by DelMar et al. [42] and on the experimental procedure described by Meissner et al. [35]. The following information was previously published by Habicher et al. [13]. The assay was carried out in a microplate reader (Synergy 4, BioTek, Winooski, VT, USA) at a wavelength of $405 \mathrm{~nm}$. The concentration of the substrate stock solution of N-succinyl-alanine-alanine-proline-phenylalanine-p-nitroanilide (N-Suc-AAPF-pNA, Bachem AG, Bubendorf, Switzerland) was $60 \mathrm{mg} / \mathrm{mL}$ using water free dimethyl sulfoxide (DMSO). Stocks were stored at $-20^{\circ} \mathrm{C}$ for not longer than 6 months. Prior to use, the substrate stock was diluted $1 / 50$ with $0.1 \mathrm{M}$ Tris $\mathrm{HCl}$ buffer, $\mathrm{pH} 8.6,0.1 \%$ (w/v) Brij 35 (reaction buffer). The reaction was started by adding $100 \mu \mathrm{L}$ of diluted substrate stock to $50 \mu \mathrm{L}$ of sample in a 96-well microtiter plate with clear and flat bottom (Rotilabo microtest plates, N${ }^{\circ}$ 293.1, Carl Roth $\mathrm{GmbH}+\mathrm{Co}$. KG, Karlsruhe, Germany). The absorption measurement was conducted at $30{ }^{\circ} \mathrm{C}$ for $15 \mathrm{~min}$. Samples were diluted with reaction buffer to keep the absorption at $405 \mathrm{~nm}$ between 0.05 and 1. Protease activity was calculated based on the change of absorption at $405 \mathrm{~nm}$, the extinction coefficient $\varepsilon$ of 8,900 $1 / \mathrm{M} / \mathrm{cm}$ and the pathlength of $0.43 \mathrm{~cm}$. Protease activities were normalized to a reference process (Fig. 4) or to control strains (Ctrl \#1 and \#2, Fig. 5). Within this 
work, protease activities are referred to as relative protease activities.

\section{Supplementary Information}

The online version contains supplementary material available at https://doi. org/10.1186/s12934-021-01541-2.

Additional file 1. Course of the scattered light intensity of a single preculture with complex TB medium and main culture with mineral salt medium. Cultures are exemplarily shown using one B. licheniformis strain. The Arabic numbers stand for single colonies that were picked from agar plates and transferred into individual wells of a FlowerPlate ${ }^{\circledR}$. Scattered light intensity in the preculture and main culture shows biomass growth over time. In the batch preculture with complex TB medium scattered light intensities reach a plateau at around $12 \mathrm{~h}$, which lasts for at least $18 \mathrm{~h}$. Thus, the batch preculture was harvested after $16 \mathrm{~h}$ of cultivation. $1 \%(\mathrm{v} / \mathrm{v})$ of the preculture filling volume, which corresponds to $8 \mu \mathrm{L}$, was transferred to the batch main culture with mineral salt medium. Cultivation conditions: FlowerPlate ${ }^{\circledR}, n=1000 \mathrm{rpm}, d_{0}=3 \mathrm{~mm}, V_{L}=0.8 \mathrm{~mL}, 30$ ${ }^{\circ} \mathrm{C}$.

Additional file 2. Batch main culture of $B$. licheniformis with mineral salt medium. The main culture was inoculated according to the procedure depicted in Fig. 3. Shadows symbolize the standard deviation of cultivations with four individually picked colonies $(n=4)$. Roman numbers divide the course of the cultivation into three distinct phases: I exponential growth phase on glucose, II growth phase on overflow metabolites, and III carbon starvation phase. Glucose depletion (dashed line between I and II) is visible in the scattered light intensity and indicated by the switch of the $\mathrm{pH}$ and dissolved oxygen tension (DOT) into an upward direction. The $\mathrm{pH}$ switch is caused by the consumption of previously accumulated overflow metabolites. Overflow metabolite consumption (II) is recognizable in the course of the scattered light intensity and the DOT. The scattered light intensity increases again whereas the DOT exhibits another downward spike. Carbon starvation (dashed line between II and III) is indicated by the DOT approaching $100 \%$ and the constantly decreasing scattered light intensity. Cultivation conditions: FlowerPlate ${ }^{\circledR}$ with $\mathrm{pH}$ and DOT optode, $n=1000 \mathrm{rpm}, d_{0}=3 \mathrm{~mm}, V_{\mathrm{L}, \text { Main culture (batch) }}=0.88 \mathrm{~mL}, 30^{\circ} \mathrm{C}$.

Additional file 3. Glucose release over time using the fed-batch MTP. a Glucose release within an individual well $(n=6)$ of the fed-batch MTP with the mineral salt medium used within this study. Cultivation conditions: $n=1000 \mathrm{rpm}, d_{0}=3 \mathrm{~mm}, V_{\mathrm{L}}=0.77 \mathrm{~mL}, 30^{\circ} \mathrm{C}$. b Glucose release within an individual well $(n=3)$ of the fed-batch MTP with V3 mineral medium. Adapted with permission from Habicher et al. (2020). In a and $\mathbf{b}$ similar fed-batch MTP's (48-round-well) were used and show a comparable release of glucose. Keil et al. (2019) provided an equation to calculate glucose release under different media and cultivation conditions in 96-square-well fed-batch MTP's.

Additional file 4. Detectable difference and statistical power as function of the number of replicates. $\mathbf{a}$ Detectable difference as function of the number of replicates. The statistical power and the standard deviation were set to 0.8 and $\pm 10 \%$, respectively. $\mathbf{b}$ Statistical power as function of the number of replicates. The detectable difference and the standard deviation were set to $\pm 15 \%$ and $\pm 10 \%$, respectively. Calculations were done with the MATLAB function sampsizepwr with a two-tailed t-test (normally distributed) as test type.

\section{Acknowledgements}

The authors thank Dr. Nina Ihling for critically revising the manuscript.

\section{Authors' contributions}

TH conducted the preliminary work for applying the fed-batch MTP's with B. licheniformis, analyzed and interpreted data and drafted the manuscript. TK designed the study, analyzed and interpreted data and critically revised the manuscript. JBE did most of the experimental work and participated in data analysis and interpretation. AD participated in data interpretation and critically revised the manuscript. JBU supervised the study, participated in data interpretation and critically revised the manuscript. All authors read and approved the final manuscript.

\section{Funding}

Open Access funding enabled and organized by Projekt DEAL. The authors thank BASF SE for funding this project.

\section{Availability of data and materials}

The data that support the findings of this study are available from BASF SE but restrictions apply to the availability of these data, which were used under license for the current study, and so are not publicly available. Data are however available from the authors upon reasonable request and with permission of BASF SE.

\section{Ethics approval and consent to participate}

Not applicable.

\section{Consent for publication}

Not applicable.

\section{Competing interests}

The authors declare that they have no competing interest.

\section{Author details}

${ }^{1}$ RWTH Aachen University, AVT - Biochemical Engineering, Forckenbeckstraße 51, 52074 Aachen, Germany. ${ }^{2}$ BASF SE, Carl-Bosch-Straße 38, 67056 Ludwigshafen am Rhein, Germany.

Received: 3 October 2020 Accepted: 10 February 2021

Published online: 23 February 2021

\section{References}

1. Gupta R, Beg Q, Lorenz P. Bacterial alkaline proteases: molecular approaches and industrial applications. Appl Microbiol Biotechnol. 2002;59:15-32

2. Maurer K-H. Detergent proteases. Curr Opin Biotechnol. 2004;15:330-4.

3. Contesini FJ, de Melo RR, Sato HH. An overview of Bacillus proteases: from production to application. Crit Rev Biotechnol. 2018;38:321-34.

4. Schallmey M, Singh A, Ward OP. Developments in the use of Bacillus species for industrial production. Can J Microbiol. 2004:50:1-17.

5. Liu X, Yang $\mathrm{H}$, Zheng J, Ye Y, Pan L. Identification of strong promoters based on the transcriptome of Bacillus licheniformis. Biotechnol Lett. 2017;39:873-81.

6. Cui W, Han L, Suo F, Liu Z, Zhou L, Zhou Z. Exploitation of Bacillus subtilis as a robust workhorse for production of heterologous proteins and beyond. World J Microbiol Biotechnol. 2018;34:145.

7. Klöckner W, Büchs J. Advances in shaking technologies. Trends Biotechnol. 2012;30:307-14.

8. Lattermann C, Büchs J. Microscale and miniscale fermentation and screening. Curr Opin Biotechnol. 2015;35:1-6.

9. Huber R, Ritter D, Hering T, Hillmer A-K, Kensy F, Müller C, et al. RoboLector-a novel platform for automated high-throughput cultivations in microtiter plates with high information content. Microb Cell Fact. 2009;8:42.

10. Duetz WA. Microtiter plates as mini-bioreactors: miniaturization of fermentation methods. Trends Microbiol. 2007;15:469-75.

11. Minihane BJ, Brown DE. Fed-batch culture technology. Biotechnol Adv. 1986:4:207-18

12. Liu L, Liu Y, Shin H, Chen RR, Wang NS, Li J, et al. Developing Bacillus spp. as a cell factory for production of microbial enzymes and industrially important biochemicals in the context of systems and synthetic biology. Appl Microbiol Biotechnol. 2013;97:6113-27.

13. Habicher T, John A, Scholl N, Daub A, Klein T, Philip P, et al. Introducing substrate limitations to overcome catabolite repression in a protease producing Bacillus licheniformis strain using membrane-based fed-batch shake flasks. Biotechnol Bioeng. 2019;116:1-15.

14. Wiegand S, Voigt B, Albrecht D, Bongaerts J, Evers S, Hecker M, et al. Fermentation stage-dependent adaptations of Bacillus licheniformis during enzyme production. Microb Cell Fact. 2013;12:120. 
15. Voigt B, Hoi LT, Jürgen B, Albrecht D, Ehrenreich A, Veith B, et al. The glucose and nitrogen starvation response of Bacillus licheniformis. Proteomics. 2007;7:413-23.

16. Tännler S, Zamboni N, Kiraly C, Aymerich S, Sauer U. Screening of Bacillus subtilis transposon mutants with altered riboflavin production. Metab Eng. 2008;10:216-26.

17. Scheidle M, Jeude M, Dittrich B, Denter S, Kensy F, Suckow M, et al. High-throughput screening of Hansenula polymorpha clones in the batch compared with the controlled-release fed-batch mode on a small scale. FEMS Yeast Res. 2010;10:83-92.

18. Eck A, Schmidt M, Hamer S, Ruff AJ, Förster J, Schwaneberg U, et al. Improved microscale cultivation of Pichia pastoris for clonal screening. Fungal Biol Biotechnol. 2018:5:1-14.

19. Hemmerich J, Adelantado N, Barrigón J, Ponte X, Hörmann A, Ferrer P, et al. Comprehensive clone screening and evaluation of fed-batch strategies in a microbioreactor and lab scale stirred tank bioreactor system: application on Pichia pastoris producing Rhizopus oryzae lipase. Microb Cell Fact. 2014;13:36.

20. Hemmerich J, Noack S, Wiechert W, Oldiges M. Microbioreactor systems for accelerated bioprocess development. Biotechnol J. 2018:13:1-9.

21. Neubauer P, Cruz N, Glauche F, Junne S, Knepper A, Raven M. Consistent development of bioprocesses from microliter cultures to the industrial scale. Eng Life Sci. 2013;13:224-38.

22. Funke M, Buchenauer A, Schnakenberg U, Mokwa W, Diederichs S, Mertens A, et al. Microfluidic biolector-microfluidic bioprocess control in microtiter plates. Biotechnol Bioeng. 2010;107:497-505.

23. Funke M, Buchenauer A, Mokwa W, Kluge S, Hein L, Müller C, et al. Bioprocess control in microscale: scalable fermentations in disposable and user-friendly microfluidic systems. Microb Cell Fact. 2010;9:86

24. Panula-Perälä J, Šiurkus J, Vasala A, Wilmanowski R, Casteleijn MG, Neubauer P. Enzyme controlled glucose auto-delivery for high cell density cultivations in microplates and shake flasks. Microb Cell Fact. 2008;7:1-12.

25. Toeroek C, Cserjan-Puschmann M, Bayer K, Striedner G. Fed-batch like cultivation in a micro-bioreactor: Screening conditions relevant for Escherichia coli based production processes. Springerplus. 2015:4:490

26. Keil T, Dittrich B, Lattermann C, Habicher T, Büchs J. Polymer-based controlled-release fed-batch microtiter plate-diminishing the gap between early process development and production conditions. J Biol Eng. 2019;13:18.

27. Habicher T, Rauls EKA, Egidi F, Keil T, Klein T, Daub A, et al. Establishing a fed-batch process for protease expression with Bacillus licheniformis in polymer-based controlled-release microtiter plates. Biotechnol J. 2020;15:1900088.

28. Huber R, Scheidle M, Dittrich B, Klee D, Büchs J. Equalizing growth in high-throughput small scale cultivations via precultures operated in fedbatch mode. Biotechnol Bioeng. 2009;103:1095-102.

29. Keil T, Landenberger M, Dittrich B, Selzer S, Büchs J. Precultures grown under fed-batch conditions increase the reliability and reproducibility of high-throughput screening results. Biotechnol J. 2019;14:1800727.
30. Huber R, Palmen TG, Ryk N, Hillmer A-K, Luft K, Kensy F, et al. Replication methods and tools in high-throughput cultivation processes-recognizing potential variations of growth and product formation by on-line monitoring. BMC Biotechnol. 2010;10:22.

31. Paul S, Aggarwal C, Thakur JK, Bandeppa GS, Khan MA, Pearson LM, et al. Induction of osmoadaptive mechanisms and modulation of cellular physiology help Bacillus licheniformis strain SSA 61 adapt to salt stress. Curr Microbiol. 2015;70:610-7.

32. Schroeter R, Hoffmann T, Voigt B, Meyer H, Bleisteiner M, Muntel J, et al. Stress responses of the industrial workhorse Bacillus licheniformis to osmotic challenges. PLoS ONE. 2013;8:1-22.

33. Gonzalez-Pastor JE, Hobbs EC, Losick R. Cannibalism by sporulating bacteria. Science (80). 2003;301:510-3.

34. Gonzalez-Pastor JE. Cannibalism: a social behavior in sporulating Bacillus subtilis. FEMS Microbiol Rev. 2010;35:415-24.

35. Meissner L, Kauffmann K, Wengeler T, Mitsunaga H, Fukusaki E, Büchs J. Influence of nitrogen source and $\mathrm{pH}$ value on undesired poly( $\gamma$-glutamic acid) formation of a protease producing Bacillus licheniformis strain. J Ind Microbiol Biotechnol. 2015;42:1203-15.

36. Kunze M, Roth S, Gartz E, Büchs J. Pitfalls in optical on-line monitoring for high-throughput screening of microbial systems. Microb Cell Fact. 2014;13:53.

37. Fujita Y. Carbon catabolite control of the metabolic network in Bacillus subtilis. Biosci Biotechnol Biochem. 2009;73:245-59.

38. Voigt B, Schweder T, Sibbald MJJB, Albrecht D, Ehrenreich A, Bernhardt J, et al. The extracellular proteome of Bacillus licheniformis grown in different media and under different nutrient starvation conditions. Proteomics. 2006;6:268-81.

39. MühImann M, Kunze M, Ribeiro J, Geinitz B, Lehmann C, Schwaneberg U, et al. Cellulolytic RoboLector-towards an automated high-throughput screening platform for recombinant cellulase expression. J Biol Eng. 2017;11:1.

40. Goddette DW, Paech C, Yang SS, Mielenz JR, Bystroff C, Wilke ME, et al. The crystal structure of the Bacillus lentus alkaline protease, subtilisin BL, at 1.4 Å resolution. J Mol Biol. 1992;228:580-95.

41. Daub A, Golabgir Anbarani A, Klein T, Freyer S, Kaeding T, Felle MF, et al. Industrial fermentation process for Bacillus using defined medium and trace element feed. WO/2020/169564. 2020

42. DelMar EG, Largman C, Brodrick JW, Geokas MC. A sensitive new substrate for chymotrypsin. Anal Biochem. 1979;99:316-20.

\section{Publisher's Note}

Springer Nature remains neutral with regard to jurisdictional claims in published maps and institutional affiliations.

Ready to submit your research? Choose BMC and benefit from

- fast, convenient online submission

- thorough peer review by experienced researchers in your field

- rapid publication on acceptance

- support for research data, including large and complex data types

- gold Open Access which fosters wider collaboration and increased citations

- maximum visibility for your research: over $100 \mathrm{M}$ website views per year

At BMC, research is always in progress.

Learn more biomedcentral.com/submissions 